\title{
Point analysis of foods by sheath-flow probe electrospray ionization/mass spectrometry (sfPESI/MS) coupled with a touch sensor
}

Kenzo Hiraoka, Stephanie Rankin-Turner, Satoshi Ninomiya, Ryo Sekine, Hiroshi Wada, Masaya Matsumura, Sachiyo Sanada-Morimura, Fukuyo Tanaka, and Hiroshi Nonami,

Osamu Ariyada

\section{Supporting Information}
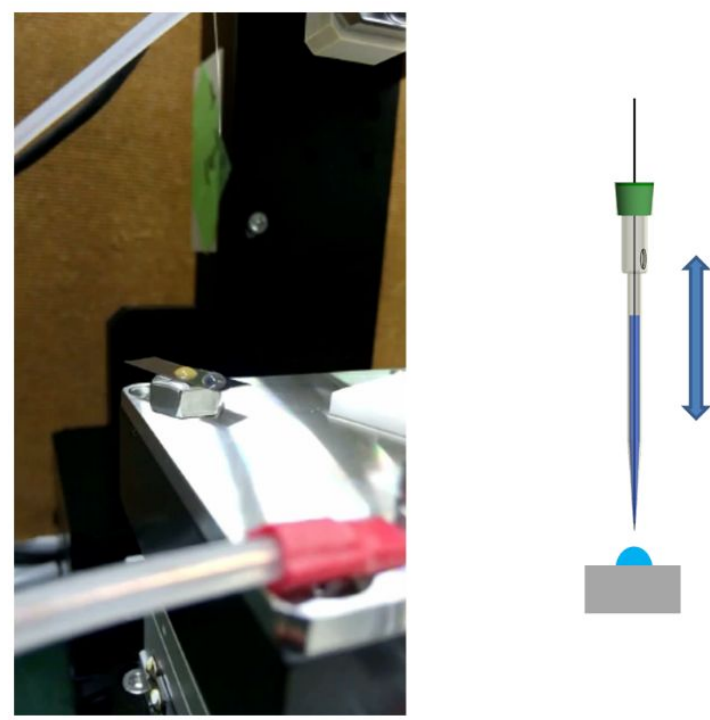

Figure S1. Video of the motion of the PESI probe equipped with a touch sensor. The invasion depth (I-D) was set to be zero. The sample was a droplet of tap water sitting on the stainless-steel sample stage.
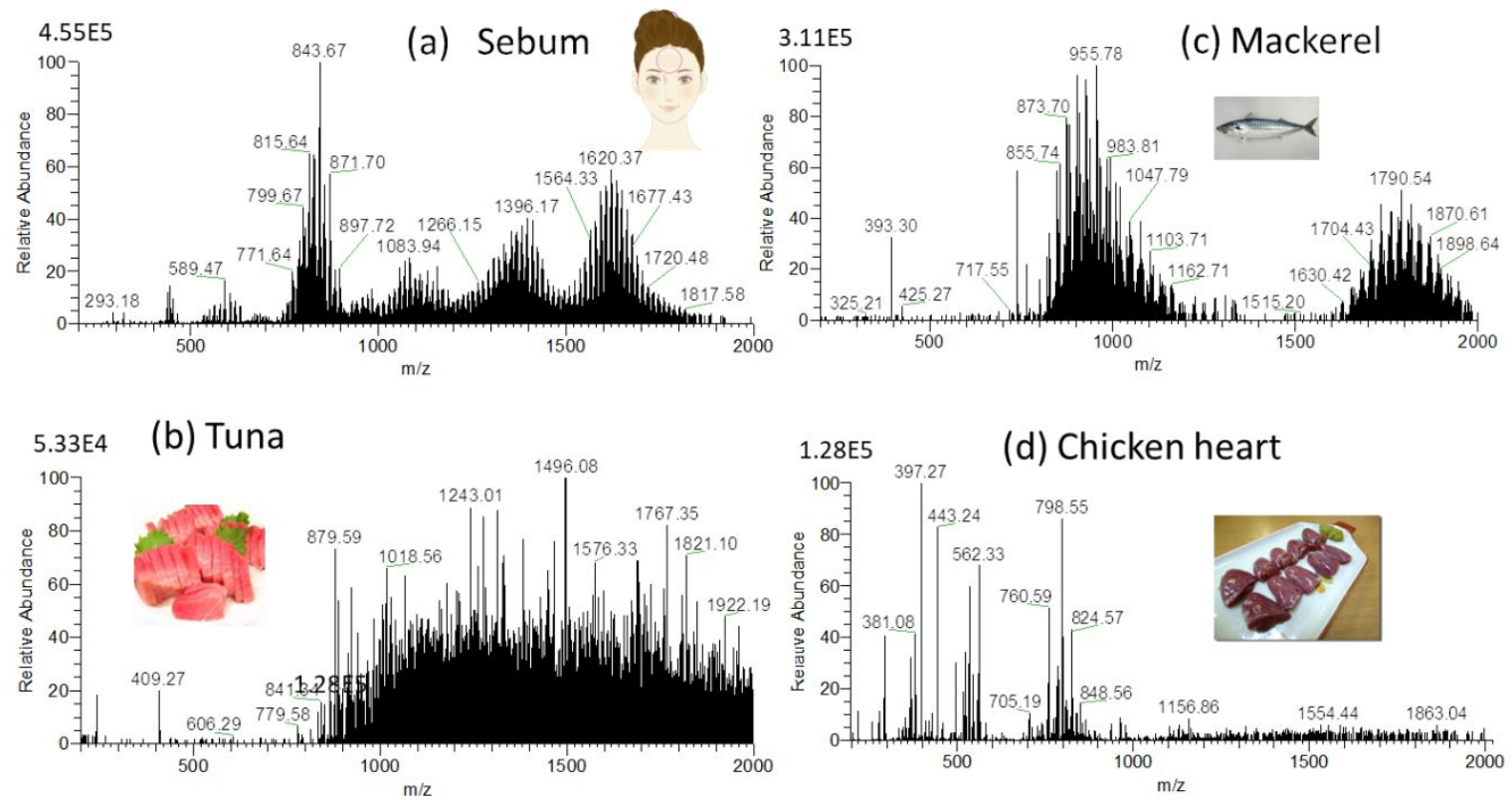

Figure S2. sfPESI mass spectra for (a) sebum taken from a forehead of a volunteer, (b) sliced tuna flesh, (c) sliced mackerel flesh, and (d) chicken heart. Sebum was pasted on the stainless steel substrate. P-L = I$\mathrm{D}=0.2 \mathrm{~mm}$. Sample extraction time: $1 \mathrm{~s}$. HV: $2.2 \mathrm{kV}$. 

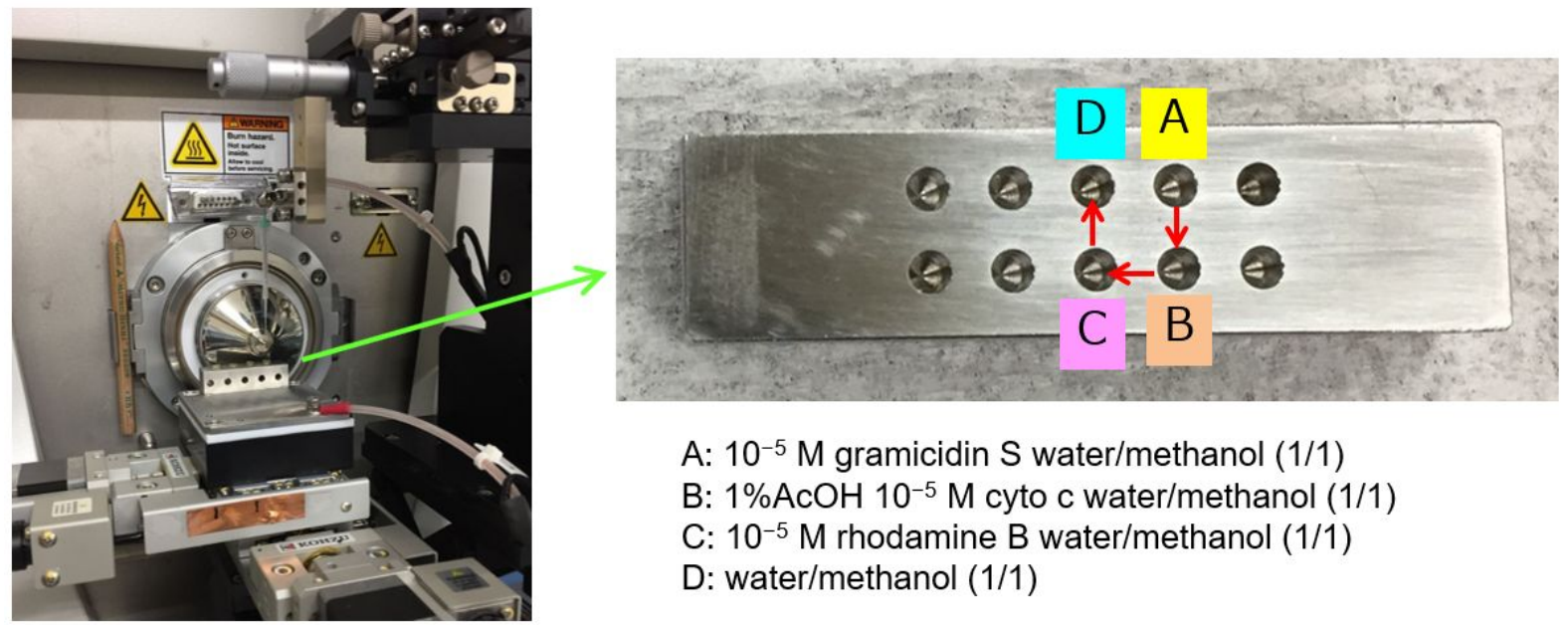
A: $10^{-5} \mathrm{M}$ gramicidin $\mathrm{S}$ water/methanol (1/1)
B: $1 \% \mathrm{AcOH} 10^{-5} \mathrm{M}$ cyto c water/methanol (1/1)
C: $10^{-5} \mathrm{M}$ rhodamine $\mathrm{B}$ water/methanol (1/1)
D: water/methanol (1/1)

Figure S3. (a) Photograph of sfPESI experimental set up. (b) The ten well stainless steel substrate. Automated measurement was made for four samples; water/methanol (1/1, v/v) solutions of (A) $10^{-5} \mathrm{M}$ gramicidin $\mathrm{S}$, (B) $1 \% \mathrm{AcOH}$ and $10^{-5} \mathrm{M}$ cytochrome $\mathrm{c}$, (C) $10^{-5} \mathrm{M}$ rhodamine $\mathrm{B}$, and (D): water/methanol (1/1) solvent. The sfPESI probe was scanned from the position from (A) to (D) as shown by the green arrows. P.L. $=0.2 \mathrm{~mm}, \mathrm{I} . \mathrm{D} .=0.2 \mathrm{~mm}$, contact time of the probe tip with the sample: $1.0 \mathrm{~s}$, duration of a HV application: $10 \mathrm{~s} . \mathrm{HV}=2.8 \mathrm{kV}$.

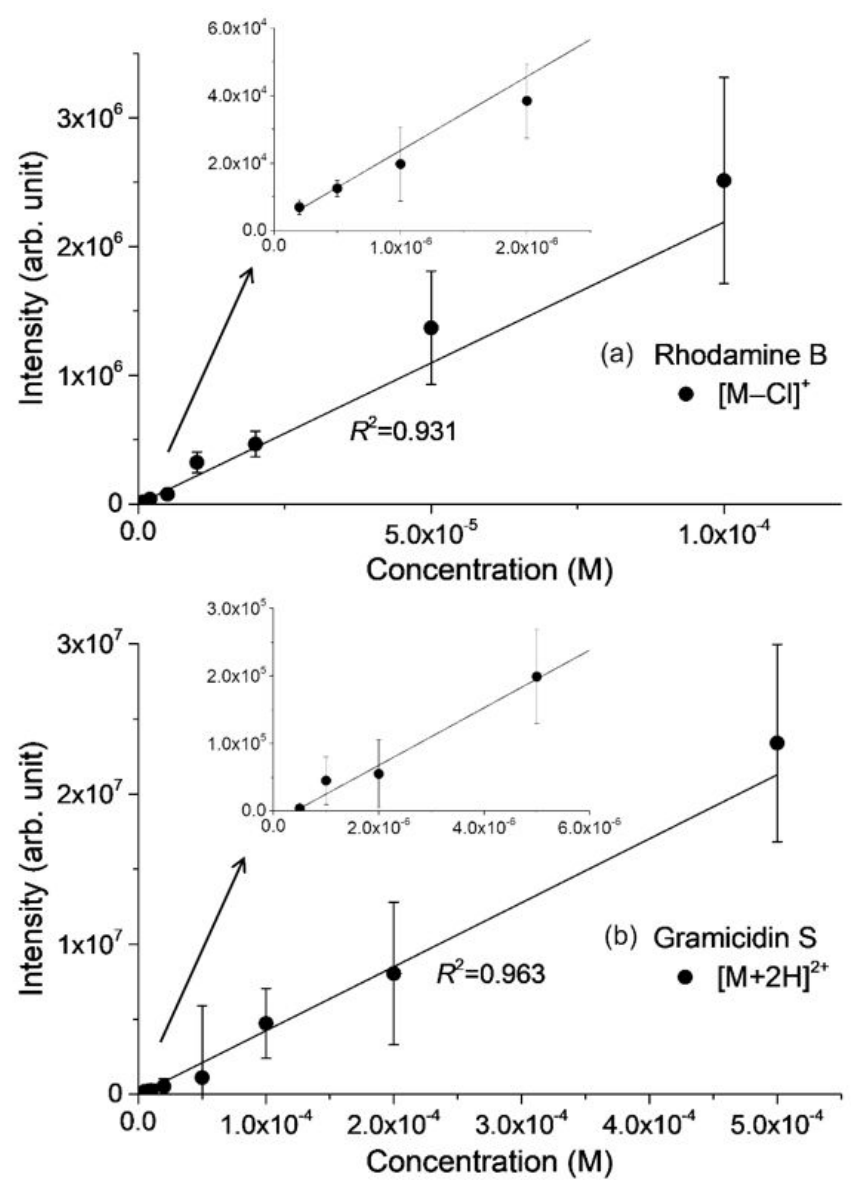

Figure S4. Relations between signal intensities and concentrations of rhodamine B (a) and gramicidin S (b). 


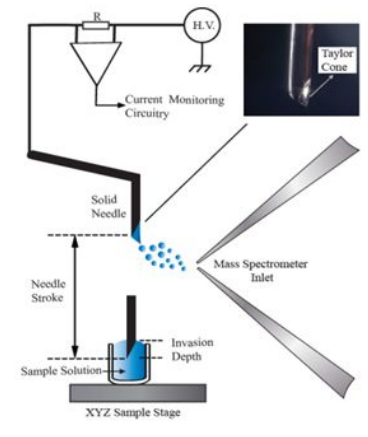

PESI

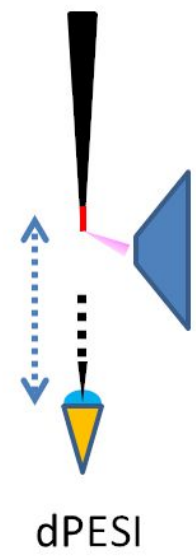

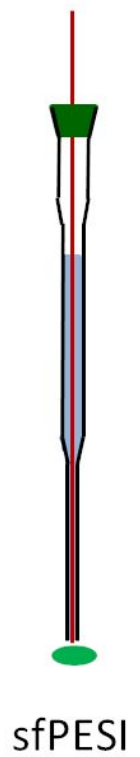

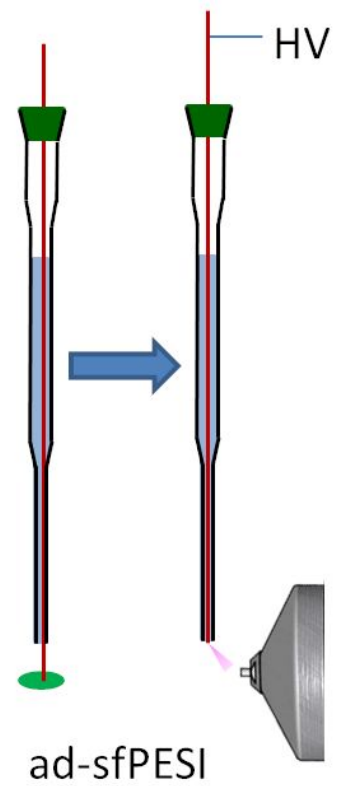

Figure S5. Conceptual ideas of PESI, dPESI, sfPESI and ad-sfPESI. 